\title{
The Framework of a Novel Approach for the Analysis of Human Movement for Clinical Purposes
}

\author{
Mark E. Williams ${ }^{1,2}$, John C. C. Williams ${ }^{3}$ \\ ${ }^{1}$ University of North Carolina, Chapel Hill, USA \\ ${ }^{2}$ Founder, LifeGait, Inc., Wilmington, USA \\ ${ }^{3}$ University of North Carolina, Wilmington DC, USA \\ Email: MEW8N@hscmail.mcc.virginia.edu
}

How to cite this paper: Williams, M.E. and Williams, J.C.C. (2017) The Framework of a Novel Approach for the Analysis of Human Movement for Clinical Purposes. Open Journal of Social Sciences, 5, 7-17. https://doi.org/10.4236/jss.2017.56002

Received: April 12, 2017

Accepted: June 6, 2017

Published: June 9, 2017

\begin{abstract}
Recent technological advances have led to the development of small wearable microelectronic sensors (accelerometers) that detect motion, gravitational acceleration, and velocity with six degrees of freedom (forward-backward, updown, and side-to-side plus rotational vectors). We have used these motion sensors to create new analytical tools called biokinetographs (BKGs). BKGs allow for more precise screening, diagnosing, monitoring, assessment and predicting of function of elderly people as they ambulate using sophisticated analysis of the unique electronic motion signature of each person. Remarkable visual differences in "functional walking signatures" are evident on the BKGs between fallers and non-fallers. This presentation will summarize our current efforts to translate this new technology into novel clinical and research tools for improving function, reducing injurious falls, and diagnosing orthopedic and neurological conditions for elderly people.
\end{abstract}

\section{Keywords}

Biotechnology, Geriatric Assessment

\section{Introduction}

The specific goal of this research is to use our patentedanalytic technology to conveniently and unobtrusively assess overall health status and well-being by way of a characteristic, qualitative biokinetic (BKG) signature. For human beings motion is a window bridging our inner and outer lives. Our movements on the purely physical plane have resonance within our inner being and reality. Likewise, our inner state is mirrored through our movements: the springing gait of optimism, the fine tremor of anxiety, or the slow shuffle of dejection. Our mo- 
tions affect and reflect our health status. In addition, immobility increases our risk of diseases such as osteoporosis, heart disease, stroke, diabetes mellitus, and possibly malignancy. Alterations in movement can result from anatomic changes (perhaps influenced by genetics), illness factors, environmental conditions and lifestyle circumstances including obesity, nutritional factors, and psychobehavioral factors such as anxiety and depression, as illustrated in Figure 1.

The interpretation of movement plays an essential role in the clinical practice of numerous medical specialties (e.g., pediatrics, sports medicine, geriatrics, physical medicine and rehabilitation, neurology, rheumatology, orthopedics, and several others). For example, the Up and Go test, in which a person (usually an elderly adult) is asked to get up out of a chair and walk across the room is rich in affective, cognitive, physical performance and diagnostic information from a geriatrician's point of view. These motion-based assessments are often communicated as subjective clinical impressions by an expert observer. Attempts to more fully explicate what the clinician perceives have captured very little of the extraordinary breadth of sensory information that is being processed during these expert evaluations. For example, efforts to objectify the Up and Go test by timing it remain unsatisfactory, because there is far more meaningful information in the process of ambulation than walking speed [1]-[9]. Accurate, precise, objective motion data provide significantly deeper insights into an individual's affective, cognitive, and physical performance status than pedometers or step counters.

LifeGait, Inc. has developed the conceptual framework, analytic strategy and biokinetic sensing devices to extend the field of motion analysis in a manner

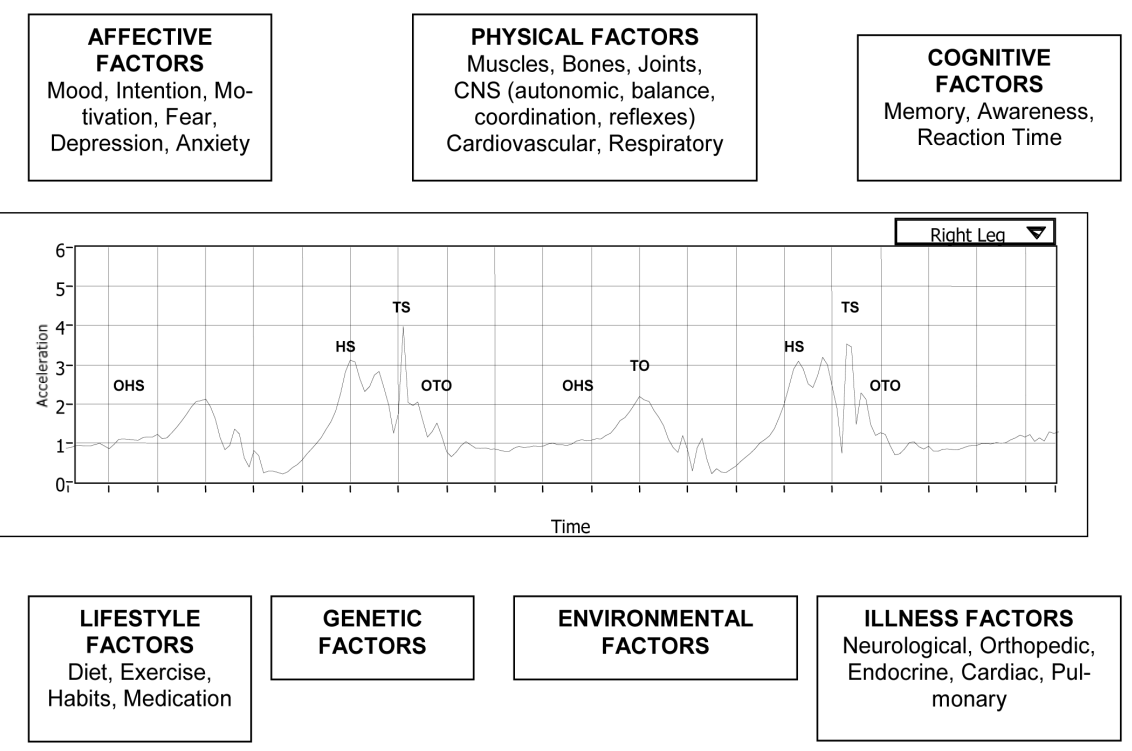

Figure 1. Biokinetic tracing (BKG) of an elderly woman showing components of the gait cycle. HS is heel strike, TS is toe strike, TO is toe off, OHS is opposite heel strike and OTO is opposite toe off. Factors shown in the boxes around the tracing may produce characteristic changes in the BKG waveforms analogous to the diagnostic utility of waveform changes seen on the EKG such as the ST segment elevation seen during a myocardial infarction. 
analogous to the way the EKG informs modern cardiology. Consider the evolution of the EKG and its impact. In 1887, British physiologist Augustus D. Waller of St Mary's Medical School, London published the first human electrocardiogram on a technician in his laboratory [10]. At the turn of the century, the EKG was viewed as an interesting clinical tool but it was impractical clinically as subjects had to place their limbs in saline solution and have the reading recorded with a galvanometer. Nonetheless the scientific community quickly recognized the potential of the new device and the value of the electrocardiogram was publicly acknowledged in 1924 when Willem Einthoven won the Nobel Prize for its invention. Since that time, innumerable patterns of electrical cardiac activity have been validated to reflect heart injury (discrete ST segment elevation), pericardial inflammation (diffuse ST segment elevation), muscle irritability (ectopic contractions), electrical malfunction (heart block), electrolyte imbalance (for example, the peaked $\mathrm{T}$ waves of hyperkalemia), hypothermia (Osbourne $\mathrm{J}$ waves), and many other physical and emotional conditions. We now have structured stress tests to evaluate one's risk for heart disease, pacemakers to restore abnormal heart rates and even implanted defibrillators to save lives from otherwise lethal arrhythmias. Heart function reflected by the EKG can be continuously monitored in the home, operating room, over the internet and even in outer space. Our task is more ambitious because we are not interested in characterizing the performance of a single organ, albeit an elegant muscular hydraulic pump. Rather, we are interested in interpreting the integrated movement patterns of the head, trunk and extremities as patterns reflective of health and illness.

Motion capture systems that provide accuracy and precision have existed for a number of years. For example, the gold standard for accessing the quality (as opposed to the quantity) of lower extremity movement evaluation (walking) is the clinical balance and gait laboratory. However, such systems possess several limitations due primarily to their cost and lack of portability. We have the analytic strategy for a portable and inexpensive motion-capture technology that emphasizes wear ability, longevity, accuracy, and precision. Refinements in digital movement-recording wearable technology can reproduce the salient measures obtained in the balance and gait laboratory, allowing ultra-sensitive, unobtrusive, continuous monitoring of human movement (including simultaneous monitoring of head, arm, waist, and leg movements) as well as characteristics of the physiologic and ambient environment such as temperature, and heart rate. These digital technologies are capable of collecting similar quality data to that provided by established motion-capture systems, but their portability and low-cost enable non-invasive and continuous motion data collection over an extended period of time and in the subjects' own environment.

Pilot studies have shown that systematic analysis of BKG waveforms can tease apart the various clinical, environmental, motivational, mental, and mechanical components and relate them to human movement. This analysis has enabled us to identify specific neurological and orthopedic conditions. Eventually we will 
develop personal movement signatures that can be gathered longitudinally to analyze the individual's age-specific performance trajectory and to develop norms and biokinetic indices for human performance analogous to the growth charts used by pediatricians to identify children with developmental abnormalities or the intelligence quotients used by psychologists. Deviations from these norms can provide an early warning of functional change before disabilities become permanent and are evident through traditional evaluations. This knowledge could be particularly valuable for concussions and sports related injuries and will stimulate new families of intervention strategies based on an improved awareness of the specific mechanisms underlying dysfunction.

\section{Our Framework}

We propose to systematically elucidate an entirely new approach to the analysis of human movement (digital biokinetics) derived from the BKG sproduced by miniature noninvasive, unobtrusive, digital sensors to understand the physical, psychological, cognitive, environmental, lifestyle and disease inputs to movement. The ultimate goal is to identify unique movement signatures that indicate either successful or unsuccessful integration of affective, cognitive, and physical

\section{INTELLECTUAL FRAMEWORK}

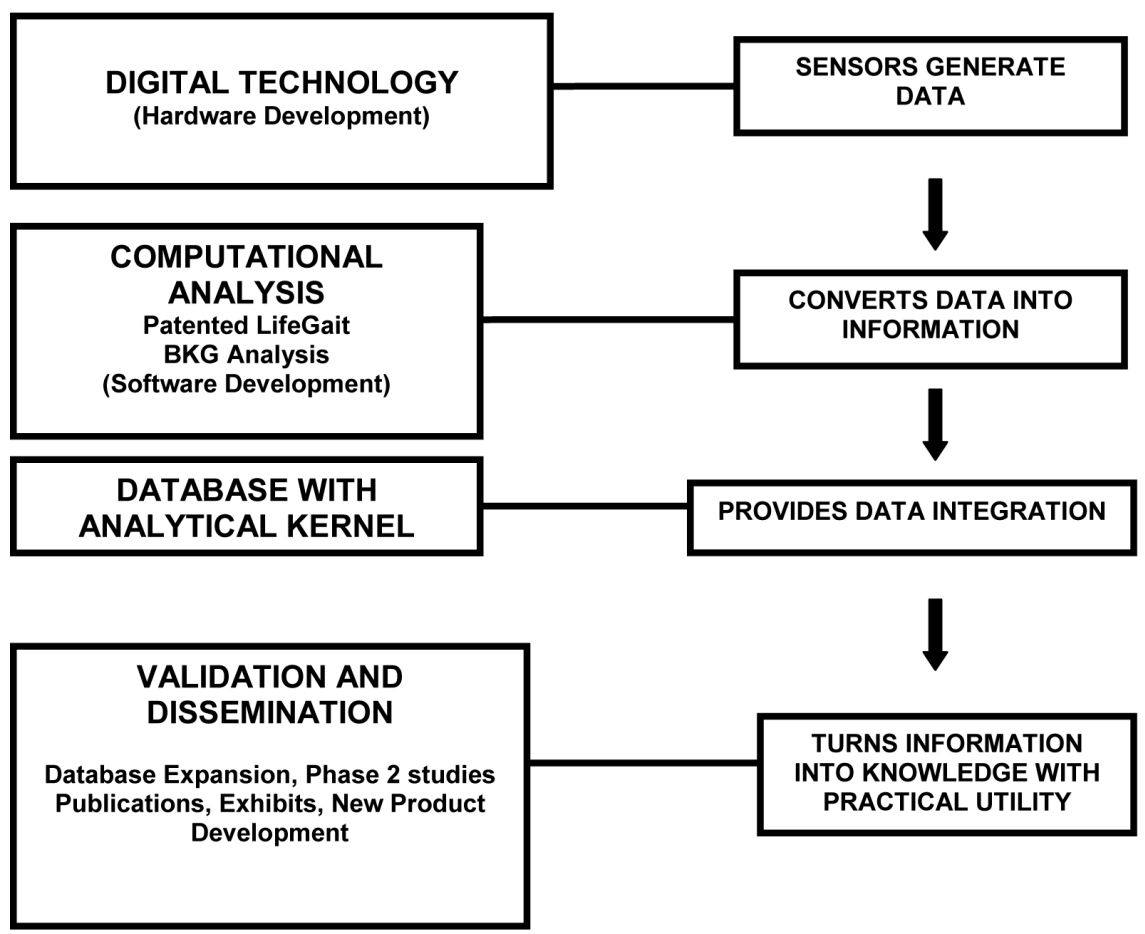

Figure 2. The intellectual framework linking the digital technology, computational analysis, the databaseand validation and dissemination. 
performance and to establish objective, ultra sensitive biomarkers of health and illness (again analogous to the utility of the waveforms on the EKG in clinical care). This will result in innovative, objective BKG measures of health, early identification of pre-disease pathways, new disease treatments, and co-morbidities assessments based, in part on individual biokinetic performance.

This new intellectual framework can provide an integrative model and sensitive metrics for the understanding and measurement of movement across the lifespan. While we can use our new technology to generate huge quantities of data on young and elderly people and their circumstances, having mountains of unrefined data is not the same as having useful information or knowledge of practical utility. We need to convert the data into useful information to assist in making helpful decisions. Moreover, the integration of the information will vary for different purposes. For example, a clinician seeing frail elderly patients will require a different data reduction strategy than the family of a healthy older person interested in monitoring health status.

\section{The Implications of This Framework}

Motion is a fundamental aspect of life. The quickening fetal movement is the first clear sign to the expectant mother that she carries new life. And at the other extreme of the life course, terminal illness is often heralded by progressive immobility. Patterns of human movement change throughout life from the uncertain steps of the toddler, to the insecure swagger of the adolescent, to the selfassured gait of responsible adulthood, and the progressive unsteadiness of geriatric frailty.

For the first time in human history, infants in fortunate nations like ours can expect to live well into their 70s. The opportunity to live into old age, once the province of the very few, is now a realistic expectation for each of us. But with this historic and unprecedented expectation of longevity comes the personal responsibility for our lifestyle choices and the impact of our decisions on our future health and function. Conversely our increasingly sophisticated information-based society is promoting a parallel change in the nature of human motion. For example, our dietary, educational, and recreational choices in childhood and adolescence may profoundly affect the nature and rate of expression of chronic illness in later life. The sedentary, fast food eater in youth may be investing in a future of chronic diseases coping with the complications of diabetes mellitus, osteoporosis and cardiovascular disease and dealing prematurely with the loss of independence [11]-[17].

The conveniences of contemporary society have substantially changed our requirements for movement. For the majority of citizens in modern societies, it is no longer necessary to walk even short distances in order to conduct all employment and daily activities, and this sedentary trend is leading to increased risk for coronary heart disease, adult-onset diabetes, hypertension, colon cancer, osteoporosis, anxiety, and depression. The profound implications of these changes in motion have been documented in a study that tracked the amount of 
daily walking performed by a cohort of Amish men and related the findings to the incidence of heart disease. Walking was quantified with simple widely available and inexpensive technology (a pedometer) that could become one of our future ongoing assessment tools. These observations strongly suggest that our health and well-being are affected not only by our participation in regular exercise, but more profoundly by the nature of our society which has facilitated a sedentary life style. The monitoring, interpretive analysis and relational assignments of movement patterns we plan to develop are meant to assist in the diagnosis, prognosis, and design of therapeutic interventions to comprehensively address just these social concerns.

Understanding the epidemiology of motion from youth through maturity is an urgent social and scientific priority. The percentage of people with disabilities severe enough to need assistance increases sharply with age: $10 \%$ for those 65 74 years old, $16.9 \%$ of those 75 - 79 years old and over $33 \%$ for those ages 80 and over. With the elderly population expected to double in size by 2040 , the sheer number of individuals facing dependency is staggering. But a person's ability to manage everyday routines cannot be determined confidently from the names of the specific diseases he or she may have or from the length of the medical problem list. For example, an octogenarian with systolic hypertension, congestive heart failure, maturity-onset diabetes mellitus, severe osteoarthritis and a past history of hip fracture might be a vigorous, independent community volunteer, or a frail, highly dependent resident of a nursing home. The systematic study of movement and activity patterns in young, middle-aged, and elderly age groups, in elite athletes, and in individuals with known chronic diseases such as Alzheimer's disease, hip osteoarthritis, or stroke will advance our systematic scientific understanding to yield insights to help us all reduce our risk of future dependency.

Established motion-capture gait laboratories (such as Video Camera Systems) have been proven to provide highly accurate motion data. However, their cost and lack of portability present major problems that make them non-conducive to the comprehensive longitudinal motion studies necessary for Digital Biokinetics. For example:

- Because a subject is being actively monitored (i.e. he/she is acutely aware of the data collection), the data collected is tainted. While helpful, the measures do not reflect normal, unconscious mobility in the subjects' own environment.

- Only a brief snapshot of overall motion is available for analysis. Variability in function even over a few minutes (analogous to the insights contained in the heart rate variability contained in the continuous rhythm strip of the EKG) cannot be assessed, much less over an extended period of time. Any longitudinal study using such technology would allow for only coarse analysis - the subject's motion at a certain date and time and the trends from a series of such collections. This is a major limitation, as it is quite likely that many interesting insights into motion and its relationship with affective, cognitive, and physical performance status may only be extracted from the fine-grained analysis provided by 
continuous data collection. In addition, continuous data collection would provide access to motion events (e.g. concussion, falls, injuries, tremors, etc.) that would likely be missed by clustered collection.

- The cost of these motion-capture systems requires that the data collection be serial. Even if a queue of subjects were constantly streaming into the laboratory (a logistical impossibility), the ability to capture data of only one subject at a time is a severe limitation.

- The laboratory evaluation is expensive, requiring additional people such as technicians to schedule, monitor, and record the assessments.

\section{Examples}

Figure 3 contrasts a healthy subject with three other subjects with clinical conditions affecting gait and who are known fallers. Remarkable visual differences in "BKG walking signatures" are evident among fallers and non-fallers.

Some movement vector components seem to be influenced by neuro-muscular factors (such as peripheral neuropathy or foot drop) and others by mechanical anatomic factors (such as previous hip fracture, osteoarthritis or amputation). In addition, psycho-behavioral conditions (anxiety and depression) seem to affect aspects of human motion as detected by these sensitive devices. These factors are of great interest with regard to their relationship to biokinetic signatures.
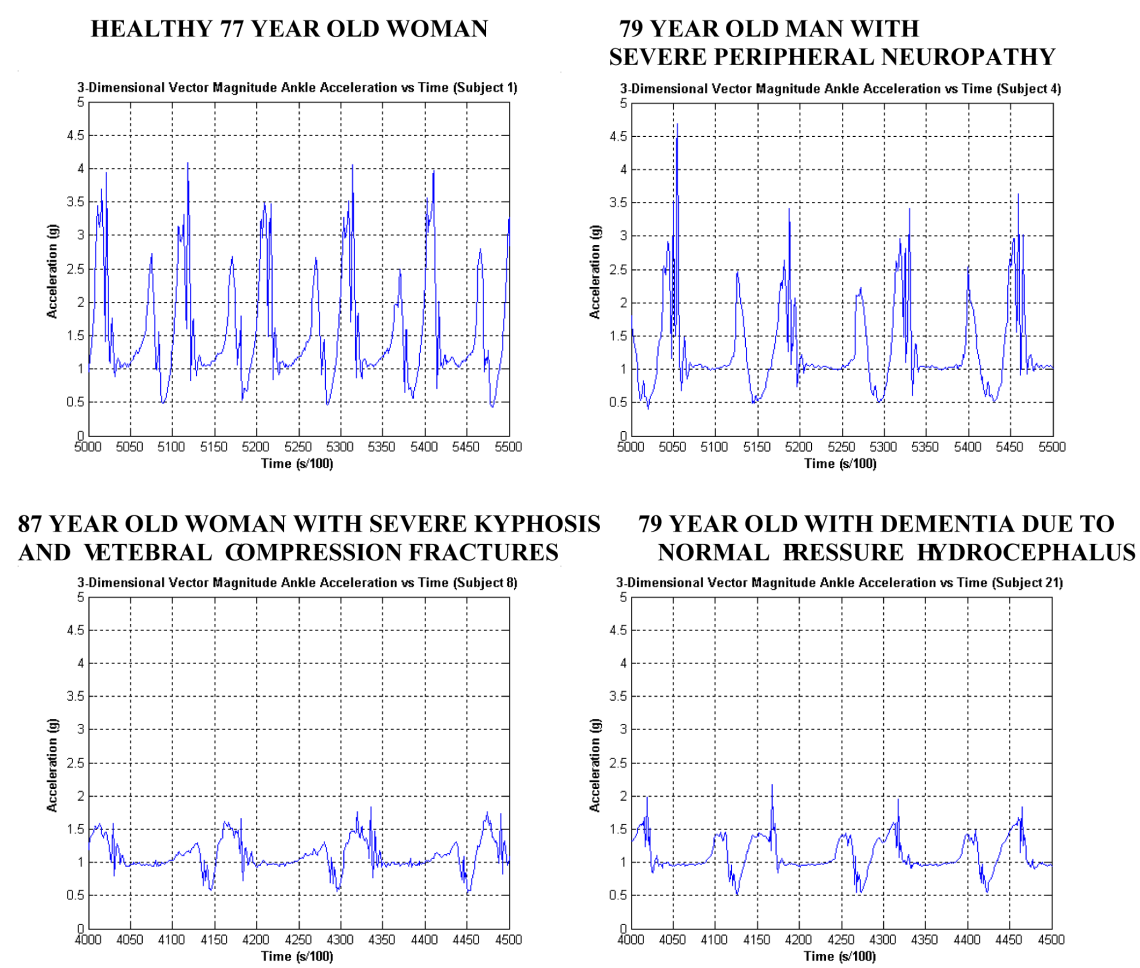

Figure 3. BKG ankle acceleration tracings for four individuals (a healthy non-faller and three fallers with differing clinical conditions). Note the extraordinary differences in the movement signatures. We quantitatively objectify these differences by implementation into intelligent interpretive analytical algorithms. 
Spectral analyses (Fast Fourier Transform) of the gait acceleration data shows that the most fit (least vulnerable elderly adults) have high peak amplitudes, representing a vigorous and steady gait. The most vulnerable subjects have much lower peak amplitudes, whereas moderately vulnerable subjects have intermediate values $(\mathrm{F}=11.7, \mathrm{p}<0.002)$. The dispersion of gait kinetic energy, as measured by the number of frequencies in the spectral analysis, also discriminates among the least, moderately and most vulnerable subjects $(\mathrm{F}=8.65, \mathrm{p}<0.006)$. The most vulnerable subjects have more than twice the number of frequencies as the least vulnerable subjects who have the lowest number of frequencies.

Figure 4 dramatically illustrates this finding. The peak amplitude of the healthy elderly adult is $0.4 \mathrm{~g}$ with a dominant peak at $2 \mathrm{~Hz}$. In strong contrast, a frail faller has peak amplitude that is 3 orders of magnitude lower and is barely visible on the same axis dimension. When the axis is adjusted so that the spectral distribution is evident, the peak amplitude at $0.6 \mathrm{Hertz}$ is much lower, at about.003g with a mounded distribution of energy centering at about 1.5 Hertz.
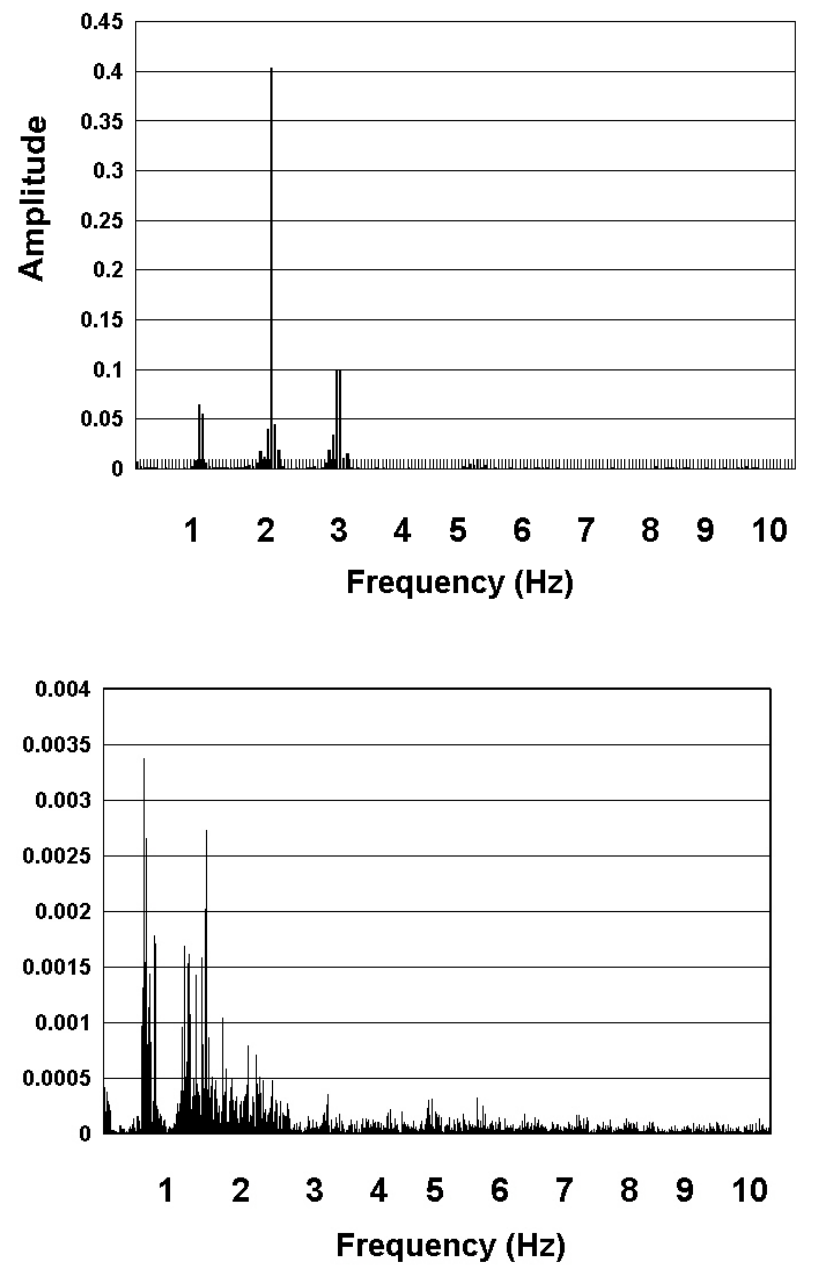

Figure 4. Spectral analysis (Fast Fourier Transforms) of gait accelerations in a normal nonfaller (left panel) and a faller (right panel). Note the remarkable amplitude differences and the scattered pattern of the faller; features expected to contribute great utility to intelligent algorithm design. 
The distribution of frequencies is much more dispersed with extremely low amplitudes and higher frequencies. So, the faller is slower and moves with far less regularity and force than the nonfaller whose movements are faster and highly regular. While simple timing with a stopwatch can reveal that the nonfalleris walking more quickly than the faller, the BKG tracing provides far more meaningful information about the quality of the motion and precise metrics for such concepts as balance, sway, consistency, steadiness and force, which are evident to the clinician observing gait but not readily quantified by clinical assessment alone.

\section{Conclusions}

We have created the BKG analytic framework for low-cost, portable motioncapture technologies that enable non-invasive and continuous motion data collection for a large number of subjects over an extended period of time. Wireless nodes distributed across the body at points of interest can store and/or transmit velocity, acceleration, torque, orientation, and position data, which can collectively be processed remotely for motion analysis. The nodes are inexpensive, unobtrusive, wearable, durable, and user-friendly, yet continuously collect accurate, precise data. All types of motion on any part of the body can be collected and analyzed, including simultaneous monitoring of head, arm, trunk, waist, and leg movements. We are also developing and integrating biological and ambient monitoring systems that can help add context to the collected motion data.

Life Gait's proprietary approach to the systematic analysis of human movement allows us to determine the effects of environmental and psychosocial challenges experienced throughout life as such as concussion; identify the affective, cognitive and physical inputs to motion in health and illness; identify individuals at risk for changes in function and independence; develop BKG indices for adult and geriatric function (analogous to the pediatrician's growth indices of height, weight, and head circumference); and discover very early motoric signs of impending diseases such as Chronic Traumatic Encephalopathy, Alzheimer's disease, Parkinson's disease, stroke, osteoarthritis of the hip and knee, and many others. Our intention is as in cardiovascular disease, for future investigators to be able to define the burden of illness; modify the specific affective, cognitive and physical risk factors for functional decline; establish programs of disability prevention; develop remedial programs for those with irreversible losses; and create various restorative strategies using motoric indices as an essential component of an individual's health profile. The need for more precise, predictive and objective markers of health status across the lifespan benefits the entire cross section of societal concerns: (1) individuals interested in health promotion, (2) families and caregivers, (3) healthcare workers, (4) hospitals, (5) long-term care institutions, (6) payers such as insurance companies, and (7) a wide variety of clinical and research settings. Effectively all facets of society will derive benefits from the success of these proposed activities. 


\section{References}

[1] Mathias, S., Nayak, U.S. and Isaacs, B. (1986) Balance in Elderly Patients: The “Get-Up and Go" Test. Arch Phys Med Rehabil, 67, 387-389.

[2] Podsiadlo, D. and Richardson, S. (1991) The Timed "Up \& Go": A Test of Basic Functional Mobility for Frail Elderly Persons. J Am Geriatr Soc, 39, 142-148. https://doi.org/10.1111/j.1532-5415.1991.tb01616.x

[3] Rose, D.J., Jones, C.J. and Lucchese, N. (2002) Predicting the Probability of Falls in Community-Residing Older Adults Using the 8-Foot Up-and-Go: A New Measure of Functional Mobility. JAPA, 10, 466-475. https://doi.org/10.1123/japa.10.4.466

[4] Thomas, J.I. and Lane, J.V. (2005) A Pilot Study to Explore the Predictive Validity of 4 Measures of Falls Risk in Frail Elderly Patients. Arch Phys Med Rehabil, 86, 1636-1640. https://doi.org/10.1016/j.apmr.2005.03.004

[5] Arnold, C.M. and Faulkner, R.A. (2007) The History of Falls and the Association of the Timed Up and Go Test to Falls and Near-Falls in Older Adults with Hip Osteoarthritis. BMC Geriatr, 7, 17-10. https://doi.org/10.1186/1471-2318-7-17

[6] Panel on Prevention of Falls in Older Persons. (2011) American Geriatrics Society and British Geriatrics Society: Summary of the Updated American Geriatrics Society/British Geriatrics Society Clinical Practice Guideline for Prevention of Falls in Older Persons. J Am Geriatr Soc, 59, 148-157. https://doi.org/10.1111/j.1532-5415.2010.03234.x

[7] Rydwik, E., Bergland, A., Forséén, L. and Fräändin, K. (2011) Psychometric Properties of Timed Up and Go in Elderly People: A Systematic Review. Physical \& Occupational Therapy in Geriatrics, 29, 102-125. https://doi.org/10.3109/02703181.2011.564725

[8] Beauchet, O., Fantino, B., Allali, G. and Muir, S.W. (2011) Montero-Odasso M, Annweiler C: Timed Up and Go Test and Risk of Falls in Older Adults: A Systematic Review. J Nutr Health Aging, 15, 933-938. https://doi.org/10.1007/s12603-011-0062-0

[9] Schoene, D., Wu, S.M., Mikolaizak, A.S., Menant, J.C., Smith, S.T. and Delbaere, K. et al. (2013) Discriminative Ability and Predictive Validity of the Timed Up and Go Test in Identifying Older People Who Fall: Systematic Review and Meta-Analysis. $J$ Am Geriatr Soc, 61, 202-208. https://doi.org/10.1111/jgs.12106

[10] Waller, A.D. (1887) A Demonstration on Man of Electromotive Changes Accompanying the Heart's Beat. J Physiol(London), 8, 229-234. https://doi.org/10.1113/jphysiol.1887.sp000257

[11] Healy, G.N., Wijndaele, K., Dunstan, D.W. et al. (2008) Objectively Measured Sedentary Time, Physical Activity, and Metabolic Risk: The Australian Diabetes, Obesity and Lifestyle Study (AusDiab). Diabetes Care, 31, 369-371. https://doi.org/10.2337/dc07-1795

[12] Dunstan, DW, Salmon, J., Healy, G.N., et al. (2007) Association of Television Viewing with Fasting and 2-H Post Challenge Plasma Glucose Levels in Adults without Diagnosed Diabetes. Diabetes Care, 30, 516-522. https://doi.org/10.2337/dc06-1996

[13] Ford, E.S., Li, C., Zhao, G., Pearson, W.S., Tsai, J. and Churilla, J.R. (2010) Sedentary Behavior, Physical Activity, and Concentrations of Insulin among US Adults. Metabolism, 59, 1268-1275. https://doi.org/10.1016/j.metabol.2009.11.020

[14] Healy, G.N., Dunstan, D.W. and Salmon, J., et al. (2007) Objectively Measured Light-Intensity Physical Activity is Independently Associated with 2-H Plasma Glucose. Diabetes Care, 30, 1384-1389. https://doi.org/10.2337/dc07-0114 
[15] Thorp, A.A., Healy, G.N., Owen, N., et al. (2010) Deleterious Associations of Sitting Time and Television Viewing Time with Cardio-Metabolic Risk Biomarkers: AusDiab 2004-2005. Diabetes Care, 33, 327-334. https://doi.org/10.2337/dc09-0493

[16] Hamburg, N.M., McMackin, C.J., Huang, A.L., et al. (2007) Physical Inactivity Rapidly Induces Insulin Resistance and Microvascular Dysfunction in Healthy Volunteers. Arterioscler Thromb Vasc Biol, 27, 2650-2656. https://doi.org/10.1161/ATVBAHA.107.153288

[17] Hamilton, M.T., Hamilton, D.G. and Zderic, T.W. (2007) The Role of Low Energy Expenditure and Sitting on Obesity, Metabolic Syndrome, Type 2 Diabetes, and Cardiovascular Disease. Diabetes, 56, 2655-2667. https://doi.org/10.2337/db07-0882

Submit or recommend next manuscript to SCIRP and we will provide best service for you:

Accepting pre-submission inquiries through Email, Facebook, LinkedIn, Twitter, etc. A wide selection of journals (inclusive of 9 subjects, more than 200 journals) Providing 24-hour high-quality service

User-friendly online submission system

Fair and swift peer-review system

Efficient typesetting and proofreading procedure

Display of the result of downloads and visits, as well as the number of cited articles Maximum dissemination of your research work

Submit your manuscript at: http://papersubmission.scirp.org/

Or contact jss@scirp.org 